\title{
Bi-modal pulsation of the $\delta$ Scuti star V1821 Cygni
}

\author{
A.-Y. Zhou, Z.-L. Liu, and B.-T. Du \\ National Astronomical Observatories, Chinese Academy of Sciences, Beijing 100012, PR China \\ Received 8 January 2001 / Accepted 15 March 2001

\begin{abstract}
The results of Johnson $V$ time-series CCD photometry of the $\delta$ Scuti star V1821 Cygni are presented. Our data set consists of 2431 differential magnitudes and spans the period of July 1999 to September 2000. We detect two best-fit pulsation frequencies representing the light variations of the variable. V1821 Cyg is most likely pulsating in a mixture of radial and nonradial modes. The uvby $\beta$ data in the existing catalogues have been used to derive the main physical parameters for the variable. We obtain: $T_{\text {eff }}=7490 \pm 200 \mathrm{~K}, M_{V}=1 .{ }^{\mathrm{m}} 02 \pm 0.2$, $\log g=3.75 \pm 0.1 \mathrm{dex}, M=2.3 \pm 0.2 M_{\odot}, R=3.3 \pm 0.3 R_{\odot}, \bar{\rho}=0.06 \pm 0.01 \bar{\rho}_{\odot}$, age $=1.0 \pm 0.1 \mathrm{Gyr}$ and a distance modulus of $\sim 99^{\mathrm{m}} 12$. V1821 Cyg is suggested to be a Population I $\delta$ Scuti star with metal-enriched abundances $([\mathrm{M} / \mathrm{H}]=0.366)$ evolving on its late main sequence stage on the basis of the colour indices and derived properties.
\end{abstract}

Key words. stars: $\delta$ Scuti stars - stars: oscillations - stars: individual: V1821 Cygni

\section{Introduction}

The decision to observe V1821 $\mathrm{Cyg}(=\mathrm{HD} 227695, \alpha=$ $20^{\mathrm{h}} 06^{\mathrm{m}} 33^{\mathrm{s}} .5, \delta=35^{\circ} 52^{\prime} 42^{\prime \prime} .0$, equinox $=2000.0, V=$ $10^{\mathrm{m}} 14, \Delta V=0{ }^{\mathrm{m}} 04$, A5p) (Rodríguez et al. 2000, hereafter R00) was taken mainly due to the poor available studies in the literature since its discovery as a $\delta$ Scuti-type variable by Delgado et al. (1984). V1821 Cyg is located in the region of cluster NGC 6871 and it is also known as H10 (Hoag et al. 1961). However, V1821 Cyg was estimated to be of spectral type A8 III and it is a foreground object and not a member of the young open cluster NGC 6871 (Delgado et al. 1984, hereafter D84). Based on three nights of observations, D84 obtained two pulsation frequencies at 9.245 and $11.076 \mathrm{~cd}^{-1}$, which could correspond to the first and second, or the second and third radial overtones. They suspected the presence of the fundamental mode in their residual periodogram and expected that this could be confirmed. In this sense, new observations were carried out in two observing seasons 1999 and 2000. We show evidence for the intrinsic bi-periodic pulsation in the light curves of V1821 Cyg and slight variability in frequency solutions indicating the likely presence of amplitude and period changes in the star.

\section{Data acquisition}

The observations were secured from 27 July 1999 to 9 September 2000. A journal of the observations is given

Send offprint requests to: A.-Y. Zhou,

e-mail: aiying@bao.ac.cn in Table 1, where the unfiltered observations are labelled "W". The $V$ data set consists of 21 nights (90.7 hours) spanning 354 days and yielded 2431 measurements.

The CCD Johnson $V$ photometry of V1821 Cyg was carried out with the CCD light-curve survey photometer mounted on the $85-\mathrm{cm}$ telescope at the Xinglong Station of Beijing Astronomical Observatory (BAO) of China. The CCD photometer used a red-sensitive Thomson TH7882 $576 \times 384 \mathrm{CCD}$ with a whole imaging size of $13.25 \times$ $8.83 \mathrm{~mm}^{2}$ corresponding to a sky field-of-view of $11.5 \times 7 ! 7$ (square arcmin), which allows sufficient stars to be toggled in a frame as reference. The magnitude differences between two comparison stars observed in the field of V1821 Cyg yielded a typical accuracy of 0.010 to $0{ }^{\mathrm{m}} 006$. One of the comparison stars, GSC 2683-3076, was discovered to be a new low-amplitude multiperiodic $\delta$ Scuti star $(\mathrm{Du}$ et al. 1999; Zhou et al. 2001a) as a by-product of the observations. No variability was detected in the light curves of GSC 2683-2994 $\left(\alpha=20^{\mathrm{h}} 06^{\mathrm{m}} 29 \mathrm{~s} .47, \delta=35^{\circ} 54^{\prime} 42^{\prime \prime} \cdot 0\right.$, 2000.0, $\left.V=10^{\mathrm{m}} 6, \mathrm{~F} 8\right)$ within the accuracy of our observations. Hence this star was finally selected as comparison to produce differential magnitudes for the variable. Differential atmospheric and colour extinction effects were eliminated whenever they appeared in the light curves of V1821 Cyg. Exposure times ranged from 10 to $60 \mathrm{~s}$ depending on the atmospheric conditions. Most of the $V$ data have been merged into 120 -s bins, the others 60 -s bins. The detailed features of the CCD system and the procedures of data reduction including on-line bias subtraction, dark reduction and flatfield correction have been described in Wei et al. (1990) and Zhou et al. (2001a). 
Table 1. Journal of CCD Johnson $V$ photometry of V1821 Cyg

\begin{tabular}{ccrcc}
\hline $\begin{array}{c}\text { Date } \\
\text { yymmdd }\end{array}$ & $\begin{array}{c}\text { HJD(start) } \\
2451000+\end{array}$ & Points & Filter & $\begin{array}{c}\text { Int. time } \\
\text { second }\end{array}$ \\
\hline 990727 & 386.0910 & 216 & $W$ & 60 \\
990728 & 387.1828 & 109 & $W$ & 60 \\
990815 & 406.1062 & 70 & $W$ & 60 \\
990816 & 407.0894 & 45 & $W$ & 60 \\
990821 & 412.0260 & 113 & $W$ & 60 \\
990920 & 441.9880 & 130 & $V$ & 60 \\
991014 & 466.0621 & 137 & $V$ & 60 \\
991015 & 467.0380 & 92 & $V$ & 120 \\
991016 & 468.0584 & 65 & $V$ & 120 \\
991017 & 468.9503 & 198 & $V$ & 60 \\
991018 & 469.9410 & 115 & $V$ & 120 \\
991019 & 470.9614 & 161 & $V$ & 60 \\
991021 & 473.0471 & 94 & $V$ & 120 \\
991024 & 475.9338 & 69 & $V$ & 120 \\
991028 & 479.9474 & 165 & $V$ & 60 \\
991029 & 480.9266 & 82 & $V$ & 120 \\
991031 & 482.9335 & 116 & $V$ & 120 \\
991114 & 497.0134 & 81 & $V$ & 60 \\
000829 & 786.0540 & 123 & $V$ & 120 \\
000831 & 788.0316 & 97 & $V$ & 120 \\
000901 & 789.0101 & 109 & $V$ & 120 \\
000905 & 793.0175 & 120 & $V$ & 120 \\
000906 & 794.0056 & 133 & $V$ & 120 \\
000907 & 795.0090 & 116 & $V$ & 120 \\
000908 & 796.0618 & 91 & $V$ & 120 \\
000909 & 796.9986 & 137 & $V$ & 120 \\
\hline & & & &
\end{tabular}

\section{Frequency analysis}

The frequency analysis was carried out by using PERIOD98 (Breger 1990; Sperl 1998) and MFA (Hao 1991; Liu 1995). We refer the reader to Zhou et al. $(1999,2001 \mathrm{~b})$ for the procedure. We detected two frequencies 8.8218 and $8.2439 \mathrm{~cd}^{-1}$ with signal-to-noise ratios $(S / N)$ over 7.0 . No additional frequencies can be identified to be intrinsic to the variable. The 2 -frequency least-squares fitting resulted in a zero point of 0 . 000392 and a standard deviation of residuals $\sigma=0{ }^{\mathrm{m}} 00616$. The fitting error is in agreement with the observational accuracy. Our calculated light curves fit the observed ones quite well. Some points where the observed light curve deviates from the calculated light curve are due largely to photometric errors. We have noted the possible systematic deviations between observations and fit on 18 October 1999 and 9 September 2000. We discarded these measurements and analysed the rest. The results are the same. The results were also checked for the subsets of data in 1999 and 2000 separately. The solutions of the three datasets are highly consistent.

Following the formulae of Montgomery \& O'Donoghue (1999) errors on the fitted frequencies, amplitudes and phases were estimated with the root-mean-square deviation of observational noise of 0.008 . The results of frequency analysis are given in Table 2. The power

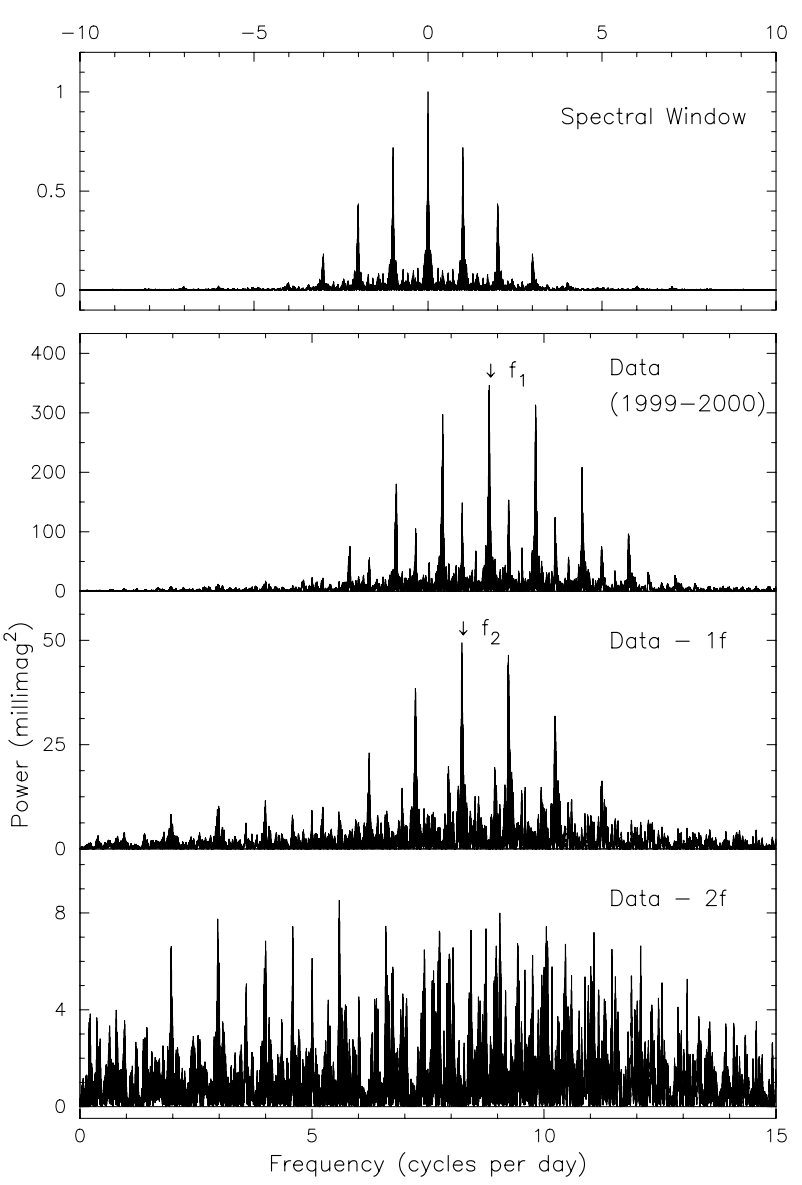

Fig. 1. The spectral window and power spectra of V1821 Cyg

spectra and spectral window are shown in Fig. 1, in which each power spectrum panel corresponds to the residuals with all the previous frequencies prewhitened. We see that the residuals in panel "Data - $2 \mathrm{f}$ " are mostly noise. The differential CCD light curves along with the fit of the 2-frequency are presented in Fig. 2. In addition to the $V$ data, we also acquired 553 unfiltered measurements ( $\sim 18$ hours) on five nights. The unfiltered light curves can be well fitted by the same two frequencies, allowing variations of amplitude and phase. Figure 3 displays the results.

The two frequencies obtained by Delgado et al. (1984) were not fully supported in our work. A reanalysis of their data showed two terms at 11.0674 and $8.9788 \mathrm{~cd}^{-1}$ with amplitudes of 0.009 and 0.0078 , respectively. The standard deviation of the residuals after prewhitening the two terms is $\sigma=0$. 0085 . We further calculated $S / N$ for the two frequencies and they are 5.1 and 4.2, respectively. If the term at $11.0674 \mathrm{~cd}^{-1}$ (near to $\nu_{2}=11.076 \mathrm{~cd}^{-1}$ by these authors) is real, it should be an unstable mode accounting for its amplitude and absence from the new data. The second term 8.9788 should be regarded as uncertain. Nonetheless, the frequency $\nu_{1}=9.245 \mathrm{~cd}^{-1}$ given by them roughly coincides with the daily alias of our frequency $f_{2}=8.2439 \mathrm{~cd}^{-1}$. Because of the limitation of their data (only 66 measurements in total), any Fourier analyses based on these data are to be less reliable and 

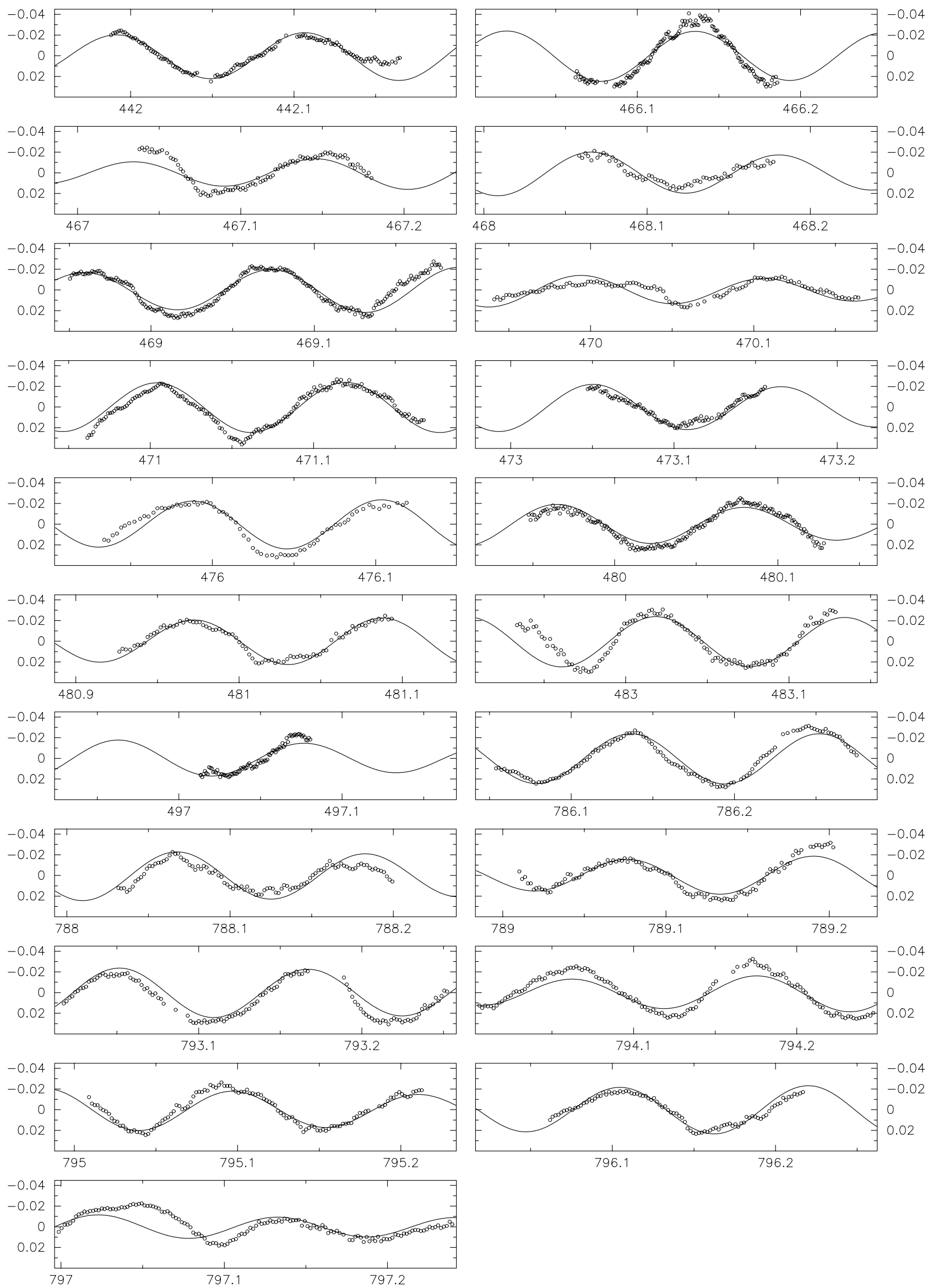

Fig. 2. $V$ differential light curves (circles) of V1821 Cyg together with the 2-frequency sinusoids represented in solid lines. Abscissa in HJD 2451000+ days, ordinate in magnitude 


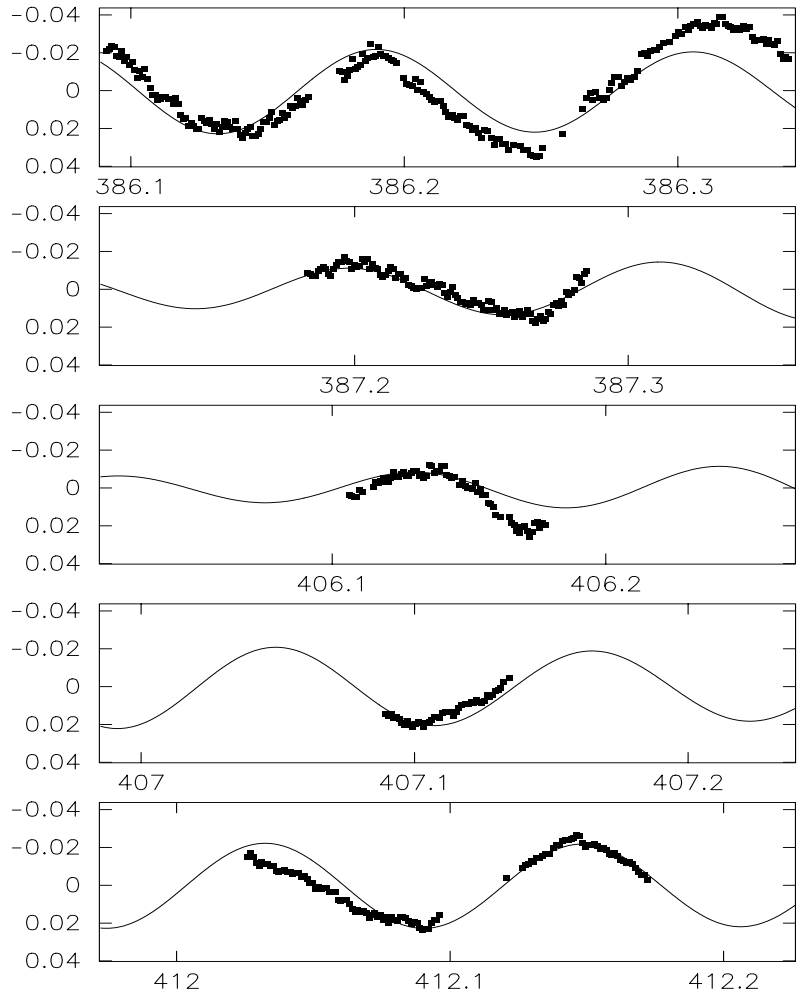

Fig. 3. The unfiltered CCD photometric magnitude differences (filled squares) of V1821 Cyg along with the 2-frequency fitted curves. Abscissa in HJD 2451000+ days, ordinate in magnitude

Table 2. Frequency contents of V1821 Cyg. Epoch relative to HJD 2451468.0+ days

\begin{tabular}{rrcccc}
\hline \multicolumn{2}{c}{$\begin{array}{c}\text { Freq. } \\
\left(\mathrm{cd}^{-1}\right)\end{array}$} & $\begin{array}{c}\text { Ampl. } \\
(\mathrm{mmag})\end{array}$ & $\begin{array}{c}\text { Phase, Epoch } \\
(0-1, \text { days })\end{array}$ & $S / N$ & $\begin{array}{c}\text { Conf. } \\
(\%)\end{array}$ \\
\hline$f_{1}$ & 8.82179 & 16.8 & $0.641, .965$ & 17.2 & 100 \\
& 2 & .2 & 14, & & \\
$f_{2}$ & 8.24389 & 7.5 & $0.875, .938$ & 7.7 & 100 \\
& 5 & .2 & 31, & & \\
\hline
\end{tabular}

must suffer from great uncertainty. We do not think their detection is real for the variable. Alternatively, we fit their light curves with our two frequencies $f_{1}=8.8218$ and $f_{2}=8.2439 \mathrm{~cd}^{-1}$ allowing variations of amplitudes and phases. Then we arrived at the amplitudes of 0.0056 and 0.0078 with $\sigma=0 .{ }^{\mathrm{m}} 0102$, which conforms to the observational accuracy of $0 .{ }^{\mathrm{m}} 013$ of the data (Delgado et al. 1984).

\section{Discussion}

\subsection{Pulsation spectrum of V1821 Cyg}

In Sect. 3, we identified what we believe to be two intrinsic modes (see Table 2). The dominating frequency at $f_{1}=8.8218 \mathrm{~cd}^{-1}$ has a semi-amplitude of $16.8 \mathrm{mmag}$. The second mode at $f_{2}=8.2439 \mathrm{~cd}^{-1}$ has a lower amplitude, but it is obviously apparent without prewhitening $f_{1}$ (see
Table 3. Amplitude variability of V1821 Cyg

\begin{tabular}{ccrr}
\hline \multirow{2}{*}{$\begin{array}{c}\text { Frequency } \\
\left(\mathrm{cd}^{-1}\right)\end{array}$} & \multicolumn{3}{c}{ Amplitude (mmag) } \\
\cline { 2 - 4 } & 1983 & 1999 & 2000 \\
\hline$f_{1}=8.82179$ & 5.6 & 16.6 & 17.0 \\
$f_{2}=8.24389$ & 7.8 & 7.5 & 7.5 \\
\hline
\end{tabular}

the second panel of Fig. 1). No frequency can be identified to be real in the residual spectrum after removing the two frequencies (see the last panel of Fig. 1). The two frequencies claimed by $\mathrm{D} 84$ were not fully verified. Their primary frequency $\left(\nu_{1}=9.245 \mathrm{~cd}^{-1}\right)$ differs from our secondary term $f_{2}=8.2439 \mathrm{~cd}^{-1}$ by $\sim 1.0 \mathrm{~cd}^{-1}$. It seemed to be the daily alias of $f_{2}$. Their $\nu_{2}=11.076 \mathrm{~cd}^{-1}$ was denied. In view of the Fourier technique, their data are too short to perform a reliable frequency analysis. In addition, D84 mentioned the possible presence of the fundamental mode in their residual periodogram. However, we saw no sign of this possibility in reanalysing their data and in our residuals after prewhitening $f_{1}$ and $f_{2}$. We are quite confident that we detected real periodicities because our data set spans a longer period of time and are of good quality.

To investigate the variability in amplitude, we performed the nonlinear least-squares fitting to the subsets of our data in 1999 and 2000 and to the data of D84 separately. The results (Table 3 ) show that the main frequency $f_{1}$ underwent an evident amplitude change from 1983 to $1999 / 2000$ but the amplitude of $f_{2}$ was constant over the period. Further observations will be necessary and helpful to study the stability of the pulsations of V1821 Cyg in amplitude as well as in period.

\subsection{Physical parameters derived from uvby $\beta$ indices}

A rough estimate of stellar physical parameters including absolute magnitude, surface gravity, effective temperature and other quantities can be derived by applying suitable calibrations for $u v b y H_{\beta}$ photometry. The values of $V, b-y, m_{1}, c_{1}$ and $\beta$ of the variable in two existing catalogues (Hauck \& Mermilliod 1998, hereafter HM98; López de Coca et al. 1990, hereafter L90) are adopted for this purpose. There is a discrepancy between the two $m_{1}$ values. According to a discussion given by Zhou et al. (2001a), the value from L90 is correct. On the other hand, we have $B-V=0.4$ and $U-B=0.18$ from R00. Applying $(B-V)$ versus $(b-y)$ colour relation of Cousins (1987) to the values, we find that $B-V=0{ }^{\mathrm{m}} 40$ corresponds to $b-y=0.249$ and $m_{1}=0.193$ if the $m_{1}$ and $b-y$ of L90 are fixed respectively. The results in turn support the values of $b-y$ and $m_{1}$ from L90.

Domingo \& Figueras (1999) showed that the Crawford (1979) calibration is able to reproduce quite well the absolute magnitude of normal A-type stars. We first dereddened the indices for the variable with the dereddening formulae and calibrations given by Crawford (1979). 
The indices are $E(b-y)=0 .{ }^{\mathrm{m}} 083,(b-y)_{0}=0{ }^{\mathrm{m}} 164$, $m_{0}=0.216, c_{0}=0.906, \delta m_{1}=-0.027$ and $\delta c_{1}=$ $0 \mathrm{~m} 206$. The results are in good agreement with those of Delgado et al. (1984) and L90.

Here we note that R00 adopted the spectral type of A5p from Reimann (1989) while Rodríguez et al. (1994, hereafter R94) used A8III for V1821 Cyg. The discrepancy in spectral type has little effect on the derived indices for the calibrations used. In addition, we also note that the two versions of the catalogues of $\delta$ Scuti stars (R94; R00) adopted the indices of $\mathrm{D} 84$, in which $\beta=2$. 720 . However, this $\beta$ value is wrong, this is a typographical mistake of D84 (Zhou et al. 2001a). The true value is $\beta=2$. 760 . This mistake was taken into account in the L90 list, but not in the catalogues of R94 nor R00.

Actually, we met a problem during derivations if $\beta=$ 2 . 720 . This $\beta$ value is the lowest limit and correspondingly $\delta c_{0}$ is larger than 0.28 , the upper limit for the Crawford (1979) calibrations. In general, late A-type stars have $\beta>2$ m 72 (i.e. $T_{\text {eff }}>7000 \mathrm{~K}$ ). $\beta=2$ m 72 seems to be lower for a star of A8III or A5p type and it is preferable to an A3-type star. In addition, Zhou et al. (2001a) obtained $\beta=2$ m 762 for GSC 2683-3076, a new $\delta$ Scuti star discovered in the field of V1821 Cyg. Both variables are foreground stars and not members of NGC 6871. Their colour indices should be very similar.

Anyway, the difference $\left(0{ }^{\mathrm{m}} 04\right)$ between the two $\beta$ values introduced an uncertainty of 0.2 for absolute magnitude. Consequently, we derived $M_{V}=1.02 \pm 0.2$ using the Crawford (1979) calibration as well as the new Strömgren photometric calibration of absolute magnitudes from HIPPARCOS (Domingo \& Figueras 1999). $\beta$ was utilized to account for effective temperature. This means a distance modulus of 9.12 , which is consistent with that of GSC 2683-3076 (Zhou et al. 2001a).

Using the adjusted $\beta, c_{0}$ grid for Kurucz model atmospheres (Moon \& Dworetsky 1985), we obtained $T_{\text {eff }}=$ $7490 \pm 200 \mathrm{~K}$ and $\log g=3.75 \pm 0.1$ dex. They agree with the estimates using the grids for $[\mathrm{Fe} / \mathrm{H}]=0.0$ or 0.5 by Smalley \& Kupka (1997) with $(b-y)_{0}$ and $c_{0}$. With $T_{\text {eff }}$ and $\log g$ and using the evolutionary tracks from Claret (1995) for solar abundances, we obtain mass $M=2.3 \pm 0.2 M_{\odot}$, age $=1.0 \pm 0.1 \mathrm{Gyr}$ and absolute bolometric magnitude $M_{\text {bol }}=1.03 \pm 0.2$ (assuming B.C. $\left.\sim 0{ }^{\mathrm{m}} 01\right)$. Then a radius of $3.3 \pm 0.3 R_{\odot}$ was calculated using the radiation law

$\log R / R_{\odot}=-0.2 M_{\mathrm{bol}}-2 \log T_{\text {eff }}+8.472$.

The results were further checked against the biparametric calibrations of stellar mass, radius and surface gravity constructed by Ribas et al. (1997).

Moreover, with $\delta m_{1}=-0{ }^{\mathrm{m}} 027$ and using the calibrations by Smalley (1993) for metallicity of A-type stars, we can obtain mean metal abundance $[\mathrm{M} / \mathrm{H}]=0.366 \mathrm{dex}$, i.e. $Z=0.046$, adopting the solar abundances $X=0.75$, $Y=0.23$ and $Z=0.02([\mathrm{Fe} / \mathrm{H}] \equiv 0.0)$ (Mazzitelli 1979). According to Stellingwerf (1979) normal $\delta$ Sct stars have solar abundances (i.e. $[\mathrm{Fe} / \mathrm{H}] \sim 0.0$ or $Z \sim 0.02$ ).
Table 4. uvby $\beta$ indices for V1821 Cyg in the existing catalogues and the dereddened values

\begin{tabular}{ccccccc}
\hline$V$ & $b-y$ & $m_{1}$ & $c_{1}$ & $\beta$ & & Ref. \\
\hline 10.122 & 0.265 & 0.099 & 0.847 & - & & HM98 \\
10.140 & 0.247 & 0.189 & 0.922 & 2.760 & & L90 \\
\hline$E(b-y)$ & $(b-y)_{0}$ & $m_{0}$ & $c_{0}$ & $\delta c_{1}$ & $\delta m_{1}$ & \\
\hline 0.082 & 0.165 & 0.215 & 0.902 & - & - & D84 \\
0.083 & 0.164 & 0.216 & 0.906 & 0.206 & -0.027 & \\
0.077 & 0.170 & 0.214 & 0.903 & 0.203 & -0.026 & L90 \\
\hline
\end{tabular}

Table 5. The dereddened indices and derived physical parameters for V1821 Cyg. D.M. stands for distance modulus

\begin{tabular}{lclc}
\hline Parameter & Values & Parameter & Values \\
\hline$E(b-y)$ & $0.083 \pm 0.02$ & $M_{V}$ & $1.02 \pm 0.2$ \\
$(b-y)_{0}$ & $0.164 \pm 0.02$ & $M_{\text {bol }}$ & $1.03 \pm 0.2$ \\
$\delta m_{1}$ & $-0.027 \pm 0.02$ & D.M. & $9.12 \pm 0.2$ \\
$\delta c_{1}$ & $0.206 \pm 0.02$ & $\log L / L_{\odot}$ & $1.49 \pm 0.08$ \\
Age $(\mathrm{Gyr})$ & $1.0 \pm 0.1$ & $T_{\text {eff }}(\mathrm{K})$ & $7490 \pm 200$ \\
$M / M_{\odot}$ & $2.3 \pm 0.2$ & $\log T_{\text {eff }}$ & $3.874 \pm 0.012$ \\
$R / R_{\odot}$ & $3.3 \pm 0.3$ & $\log g($ dex $)$ & $3.75 \pm 0.1$ \\
$\bar{\rho}_{\star} / \bar{\rho}_{\odot}$ & $0.06 \pm 0.01$ & {$[\mathrm{M} / \mathrm{H}]($ dex $)$} & 0.366 \\
\hline
\end{tabular}

Therefore the star is about 130 per cent metal-enriched compared to the Sun. The metallicity $[\mathrm{M} / \mathrm{H}]=0.366$ is also higher than $[\mathrm{M} / \mathrm{H}]=0.155$ of its neighbour star GSC 2683-3076. But the metallicity inferred from $\delta m_{1}$ is a normal value compared with $[\mathrm{Fe} / \mathrm{H}]=0.49 \pm 0.04$, the metal content of $\delta$ Scuti itself, the prototype of the $\delta$ Scuti class (Russell 1995). It is also typical of normal Population I stars. Consequently, we consider V1821 Cyg to be a metalrich Pop I $\delta$ Sct star. Table 4 summarizes the indices, comparing with those in the literature. The key stellar physical parameters for V1821 Cyg are given in Table 5. According to the colour indices and Fig. 8 of Rodríguez \& Breger (2001), the variable is well-situated in the middle of $\delta$ Scuti instability region in the Hertzsprung-Russell diagram.

\subsection{Possible mode identification}

The observational mode identifications may be roughly estimated according to the pulsation constants. Hence the physical parameters derived above (Table 5) were used to compute the observed pulsation constants $Q_{i}$ related to each frequency through the empirical formula

$\log Q_{i}=-6.456+\log \Pi_{i}+0.5 \log g+0.1 M_{\mathrm{bol}}+\log T_{\mathrm{eff}}$

given by Petersen \& Jørgensen (1972). The constant, -6.456 is based on solar values of $M_{\mathrm{bol}}=4$. 75 , B.C. $=$ $-0 .{ }^{\mathrm{m}} 08, T_{\text {eff }}=5770 \mathrm{~K}$ and $\log g=4.44$. Interpolating between the $Q$ values for stars of 2.0 and $2.5 M_{\odot}$ from Fitch (1981) results in estimates of the modes for the two frequencies. $f_{1}$ likely pulsates in nonradial $p_{1}$ mode with $l=1$. With the $Q_{2}=0 \mathrm{~d} 0302$, in the sense of the empirical period-luminosity-colour relations (Stellingwerf 1979; 
Table 6. Pulsation constants and possible modes for the frequencies detected in V1821 Cyg

\begin{tabular}{crcc}
\hline \multicolumn{2}{c}{ Frequency } & $Q$ & Modes \\
$\left(\mathrm{cd}^{-1}\right)$ & $(\mu \mathrm{Hz})$ & $($ days $)$ & \\
\hline$f_{1}=8.82179$ & 102.10 & 0.0282 & $p_{1}$ or $p_{2}(l=1)$ \\
$f_{2}=8.24389$ & 95.42 & 0.0302 & $\mathrm{~F} ; 1 \mathrm{H}$ or $p_{2}(l=1)$ \\
\hline
\end{tabular}

López de Coca et al. 1990), the corresponding mode for $f_{2}$ in radial pulsation has to be the fundamental $(\mathrm{F})$ or first overtone $(1 \mathrm{H})$. Breger et al. (1999) indicated that if the $Q$ values are calculated from $u v b y \mathrm{H}_{\beta}$ photometry, the observational uncertainties in these parameters lead to an uncertainty in $Q$ of about $18 \%$. It is of order 0.005 days (Lampens et al. 1999). Thus, mode identification might be affected by the $Q$ values.

On the other hand, the higher value of period ratio $\Pi_{1} / \Pi_{2}=0.93$ allows us to rule out the presence of a purely radial pulsation and indicates that at least one of the two modes must be nonradial. In Sect. 1, we mentioned the estimations of modes for the oscillations in Delgado et al. (1984). Now we are clear they incorrectly resolved the pulsation in the star due to insufficient data. Ultimately, for pulsation modelling, we may use the $2.1 M_{\odot}, Z=0.02$ and $2.4 M_{\odot}, Z=0.06 \bmod -$ els of Templeton et al. (1997). The two models suggest $f_{1}$ as a nonradial $p_{2}$ mode with $l=1$ and $f_{2}$ as the second radial overtone $(2 \mathrm{H})$ or a nonradial $p_{2}(l=1)$ mode. To summarize, we list the results of mode identifications in Table 6 . The attempts at mode identification at the present time are completely preliminary, due to the lack of Strömgren colour or spectroscopic data. Safe mode identification strongly needs additional colour photometry.

By means of the basic pulsation equation, the mean density and pulsation mass of the variable can be calculated in terms of its principal period and pulsation constant. With $Q_{1}=0$ d 0282 and $\Pi_{1}=0$. 1134 we find $\bar{\rho}_{\star}=0.061 \bar{\rho}_{\odot}\left(0.062\right.$ if $Q_{2}=0.0302$ and $\Pi_{2}=0.1213$ were used). The density agrees closely with the value of $0.059 \bar{\rho}_{\odot}$ derived from the Eq. (3) of Viskum et al. (1998). Then we obtained the pulsation mass $M_{\text {pul }}=2.19 M_{\odot}$ with $R=3.3 R_{\odot}$, which agrees quite well with the evolutionary mass estimated above and with that derived from the relation

$\log M / M_{\odot}=12.502+\log g-0.4 M_{\mathrm{bol}}-4 \log T_{\mathrm{eff}}$

(Petersen \& Jørgensen 1972).

\section{Conclusion}

We have collected 2431 Johnson $V$ photometric time-series measurements for the $\delta$ Sct star V1821 Cyg. They were obtained with the BAO CCD light-curve survey photometer on the $85-\mathrm{cm}$ telescope at the Xinglong Station of BAO from 27 July 1999 to 9 September 2000. Based on these data, we have investigated the pulsational behaviour for
V1821 Cyg and unambiguously identified it to be a bimodal pulsator. The light variations of V1821 Cyg can be well reproduced with the two oscillation frequencies $f_{1}=8.8218$ and $f_{2}=8.2439 \mathrm{~cd}^{-1}$. These two frequencies were detected with high $S / N$ values and are fully reliable. By making use of the uvby $\beta$ indices in the literature, the stellar physical parameters of V1821 Cyg were derived and enhanced with some discussions. The period ratio $\Pi_{1} / \Pi_{2}=0.1134 / 0.1213=0.93$ rules out the existence of two radial modes. Their corresponding $Q$ values and the comparison with the available theoretically computed pulsation models suggest that the primary frequency $f_{1}$ can be identified as a $p_{1}$ or $p_{2}$ nonradial mode of order $l=1$, while the second frequency $f_{2}$ may be the fundamental, first overtone radial mode or a $p_{2}(l=1)$ nonradial mode. V1821 Cyg probably pulsates in a mixture of radial and nonradial modes. Nevertheless, the mode identification for the detected frequencies is premature due to the lack of colour photometric data for the time being. In view of the photometric indices and our derived properties for the star, we can safely conclude that V1821 Cyg is a Population I $\delta$ Sct star with $\sim 1.3$ times solar metal abundances evolving on its late main sequence stage. However, the pulsational behaviour of V1821 Cyg deserves further intensive studies. When additional data, in particular, Strömgren photometric time series measurements, are available in the future, our knowledge on both pulsation constituents and modes of the variable will be increased. The investigation of possible causes of the exchange of power between modes, if present, and the implication for them is needed, because variability in amplitudes and periods of pulsation seems to be occurring.

Acknowledgements. We thank Prof. R.-X. Zhang and Dr. X.-B. Zhang for their joint observations. The authors are very grateful to the referee Dr. Eloy Rodríguez at IAA (Spain) for his constructive comments on the original manuscript. This research was funded by the Natural Science Foundation of China.

\section{References}

Breger, M. 1990, Comm. Asteroseismology 20, 1, Vienna: Austrian Academy of Sciences

Breger, M., Pamyatnyhk, A. A., Pikall, H., \& Garrido R. 1999, A\&A, 341, 151

Claret, A. 1995, A\&AS, 109, 441

Cousins, A. W. J. 1987, The Observatory, 107, 80

Crawford, D. L. 1979, AJ, 84, 1858

Delgado, A. J., Alfaro, E. J., García-Pelayo, J. M., et al. 1984, A\&AS, 58, 447 (D84)

Domingo, A., \& Figueras, F. 1999, A\&A, 343, 446

Du, B.-T., Zhou, A.-Y., Zhang, X.-B., et al. 1999, IBVS, No. 4805

Fitch, W. S. 1981, ApJ, 249, 218

Hao, J.-X. 1991, Publ. Beijing Astron. Obs., 18, 35

Hauck, B., \& Mermilliod, M. 1998, A\&AS, 129, 431 (HM98)

Hoag, A. A., Johnson, H. L., Iriarte, B., et al. 1961, Publ. U. S. Nav. Obs., 17, 343

Lampens, P., Van Camp, M., \& Sinachopulos, D. 1999, Delta Scuti Star Newsletter (Vienna), 13, 10 
Liu, Z.-L. 1995, A\&AS, 113, 477

López de Coca, P., Rolland, A., Rodríguez, E., \& Garrido, R. 1990, A\&AS, 83, 51 (L90)

Mazzitelli, I. 1979, A\&A, 79, 251

Montgomery, M. H., \& O'Donoghue, D. 1999, Delta Scuti Star Newletter (Vienna), 13, 28

Moon, T. T., \& Dworetsky, M. M. 1985, MNRAS, 217, 305

Petersen, J. O., \& Jørgensen, H. E. 1972, A\&A, 17, 367

Reimann, H. G. 1989, Astr. Nachr., 310, 273

Ribas, I., Jordi, C., Torra, J., \& Giménez, A. 1997, A\&A, 327, 207

Rodríguez, E., \& Breger, M. 2001, A\&A, 366, 178

Rodríguez, E., López-González, M. J., \& López de Coca, P. 2000, A\&AS, 144, 469 (R00)

Rodríguez, E., Lopez de Coca, P., Rolland, A., et al. 1994, A\&AS, 106, 21 (R94)
Russell, S. C. 1995, ApJ, 451, 747

Smalley, B. 1993, A\&A, 274, 391

Smalley, B., \& Kupka, F. 1997, A\&A, 328, 349

Sperl, M. 1998, Comm. in Asteroseismology (Vienna), 111, 1

Stellingwerf, R. F. 1979, ApJ, 227, 935

Templeton, M. R., McNamara, B. J., Guzik, J. A., et al. 1997, AJ, 114, 1592

Viskum, M., Kjeldsen, H., Bedding, T. R., et al. 1998, A\&A, 335,549

Wei, M.-Z., Chen, J.-S., \& Jiang, Z.-J. 1990, PASP, 102, 698

Zhou, A.-Y., Rodríguez, E., Jiang, S.-Y., et al. 1999, MNRAS, 308,631

Zhou, A.-Y., Rodríguez, E., Liu, Z.-L., \& Du, B.-T. 2001a, MNRAS, revised

Zhou, A.-Y., Rodríguez, E., Rolland, A., \& Costa, V. 2001b, MNRAS, in press 